\title{
Video gaming addiction and its association with memory, attention and learning skills in Lebanese children
}

\author{
Youssef Farchakh'1, Chadia Haddad ${ }^{2,3}$, Hala Sacre ${ }^{4}$, Sahar Obeid ${ }^{2,4,5^{*}+}$, Pascale Salameh ${ }^{4,6,7 \dagger}$ \\ and Souheil Hallit ${ }^{1,4^{*}+}$ (1)
}

\begin{abstract}
Background: Examining whether any association exists between addiction to video games and cognitive abilities in children could inform ongoing prevention and management of any possible harm. The objective of this study was to investigate the associations between addiction to video games, and memory, attention and learning abilities among a sample of Lebanese school children.
\end{abstract}

Methods: This cross-sectional study, conducted between January and May 2019, enrolled 566 school children aged between 9 and 13 years. Three private schools were chosen conveniently for this study. Students were randomly chosen from the list given by the school administration. The students' parents are those who responded to the questionnaire.

Results: The results showed that higher addiction to video gaming salience was significantly associated with worse episodic memory, problem solving, basic reading skills, written expression skills and worse clinical attention. Higher addiction to video gaming tolerance were significantly associated with worse novel problem solving and worse attention. Higher addiction to video gaming withdrawal were significantly associated with worse attention, factual memory, attention, processing speed, visual spatial organization, sustained sequential processing, working memory, novel problem solving and worse written expression skills.

Conclusion: The results suggest a correlation between addiction to video games and worse memory, attention, as well as cognitive and academic abilities among school children. Those findings indicate the need for more extensive research, and serve to highlight vital next steps needed in future papers, such as identifying predicting factors that could aid in early detection of video gaming addiction in children.

Keywords: Video gaming, Addiction, Learning, Attention, Memory

\footnotetext{
*Correspondence: saharobeid23@hotmail.com; souheilhallit@hotmail.com 'Sahar Obeid, Pascale Salameh, and Souheil Hallit are last co-authors

1 Faculty of Medicine and Medical Sciences, Holy Spirit University of Kaslik (USEK), Jounieh, Lebanon

${ }^{2}$ Research and Psychology Departments, Psychiatric Hospital of the Cross, P.O. Box 60096, Jal Eddib, Lebanon

Full list of author information is available at the end of the article
}

\section{Background}

Recent advances in new technologies have made video games the top leisure time occupation for children, who are particularly susceptible for addiction. Currently, video games have become the most famous type played worldwide among children. Nielsen reports that total weekly time spent playing games increased expeditiously from 5.1 to $6.3 \mathrm{~h}$ in 2011 and 2013 respectively [1]. In a study involving children aged between 9 and 12, from 12

(c) The Author(s) 2020. This article is licensed under a Creative Commons Attribution 4.0 International License, which permits use, sharing, adaptation, distribution and reproduction in any medium or format, as long as you give appropriate credit to the original author(s) and the source, provide a link to the Creative Commons licence, and indicate if changes were made. The images or other third party material in this article are included in the article's Creative Commons licence, unless indicated otherwise in a credit line to the material. If material is not included in the article's Creative Commons licence and your intended use is not permitted by statutory regulation or exceeds the permitted use, you will need to obtain permission directly from the copyright holder. To view a copy of this licence, visit http://creativeco mmons.org/licenses/by/4.0/. The Creative Commons Public Domain Dedication waiver (http://creativecommons.org/publicdomain/ zero/1.0/) applies to the data made available in this article, unless otherwise stated in a credit line to the data. 
different countries, it was found that $8.6 \mathrm{~h}$ per day were spent on playing video games [2]. Importantly, the emerging phenomenon of video game addiction represents a real and potential widespread problem that defies easy solutions and prevention methods, and requires further investigations, especially in the childhood population.

In fact, despite the benefits that video games have, such as socialization and entertainment, empirical and clinical studies have frequently demonstrated that the excessive use of video games may drive to adverse consequences in miscellaneous areas of psychological development and can result in an addiction among a small portion of gamers [3]. Impaired control over gaming and increasing priority over daily activities and other life interests could manifest as an evidence of gaming addiction [4]. In its fifth edition, the Diagnostic and Statistical Manual of Mental Disorders (DSM-5) identified video game addiction in the form of internet gaming disorder, as a condition in need of supplementary studies [5]. Additionally, the 11th revision of International Classification of Diseases (ICD-11) defined gaming disorder as a recurrent gaming behavior pattern that carries both offline and online gaming [6]. Science suggests that addictions arise from a mixture of a genetic predisposition and a repeated exposure to a particular substrate [7].

Along with that, there has been growing public concerns about potential adverse effects, including the possibility that video games might affect memory in children [8]: as an important process for comprehension, memory updating and working memory, among other cognitive skills, have usually been reported to be damaged across different behavioral disorder individuals and addictive populations [9]. Even though there are some studies that investigate the effect of video games on cognitive functions and academic performance in children [10, 11], consequences on memory are still a prevalent subject of debate.

Although video gaming is a free time activity, it does cause problems for some children, affecting their attention competencies. Both a meta-analysis and a systematic review conducted by Ho et al. and Carli et al. respectively suggested an association between inattention and internet/gaming addiction [12]. Moreover, plenty of other studies supported this finding and proved a strong correlation between the severity of inattention in attention deficit hyperactivity disorder (ADHD) and internet/gaming addiction [13], even after regulating the effects of depression and anxiety symptoms, as well as personality traits [12].

Unsurprisingly, video gaming advancements have led to concerns from parents and educators that the exaggerated amount of time consumed by children on video games, may result in decreased learning and academic abilities [11]. Handful of studies have generated inconclusive results regarding a possible association between poor school grades and problematic gaming [11, 14]. Furthermore, in cross-sectional studies, pathological video gaming has been connected to a range of negative consequences, such as lower school and academic performances, but the need for further research to explore this finding is mandatory [11]. Moreover, researchers have been attempting to shed light on addiction to video games and its association with children's development and behaviors. Despite all the efforts made, the literature still lacks sufficient studies on the subject.

In Lebanon, adolescents showed a $43.6 \%$ of moderate to severe problematic internet use [15]. Children are affected by video gaming and its related addiction. According to a Lebanese research study published in 2018, gaming disorder was associated with being younger. Moreover, Lebanese children are witnessing devastating long-term adverse effects on their behavioral, physical and psychological health, due to the rapidly advancing technology, which makes it very essential to focus on this particular Lebanese age group [14]. From the research point of view, only three Lebanese studies were detected concerning video games addiction [14, 16, 17]. Two of them worked on the development and validation of specific scales for video games addiction and internet gaming disorder $[15,16]$, while the third one explored the relationships between gaming disorders, sleep habits, and academic achievement in Lebanese adolescents [14]. The last study showed that the pooled prevalence of internet gaming disorder was 9.2\% [14]. Examining whether any association exists between addiction to video games and cognitive abilities in children could inform ongoing prevention and management of any possible harm. The objective of this study was to investigate the associations between addiction to video games, and memory, attention and learning abilities among a sample of Lebanese schoolchildren.

\section{Methods \\ Participants}

This study was a cross-sectional, conducted between January and May 2019. Three private schools were chosen conveniently for this study. Students were randomly chosen from the list given by the school administration. All participants between the age of 9 and 13 years of age were eligible to participate. The students' parents are those who answered the questionnaire. Prior to participation, parents were briefed on the study objectives and methodology, and were assured of the anonymity of their participation. Parents had the right to accept or refuse participation in the study, with no financial compensation provided in return. 


\section{Sample size calculation}

The Epi info program [Centers for Disease Control and Prevention (CDC), Epi Info ${ }^{\mathrm{TM}}$ ] was employed for the calculation of the required sample size for our study, with a prevalence of internet gaming disorder of $9.2 \%$ among 524 Lebanese high school students from a study done by Hawi et al. [14], with an acceptable margin of error of $5 \%$ and design effect of 2 . The youth population is estimated to be 585,000 according to the UNDP statistics in Lebanon; the result showed that the biggest required sample size is 256 participants.

\section{Procedure}

The questionnaire was distributed to each student in the classroom to be taken home. Parents filled it within 25 min approximately. The completed questionnaires were collected back and sent for data entry. During the data collection process, the anonymity of the participants was guaranteed.

\section{Questionnaire}

The self-administered questionnaire used was in Arabic, the native language of Lebanon. The first part assessed the sociodemographic details of the participants (i.e. age, gender, grade, and father and mother education level). The second part of the questionnaire included the following scales:

\section{Game Addiction Scale for Children (GASC)}

The 21-item Gaming Addiction Scale (GASC) is an instrument based on DSM criteria to assess gaming addiction. The seven items in the GAS are rated using a five-point Likert scale ranging from 1 (never) to 5 (very often). A higher score on the GAS indicates more problematic use of online gaming. The scale measures $7 \mathrm{cri}-$ teria of computer addiction: salience, tolerance, mood modification, withdrawal, relapse, conflict and problems [18]. In this study, the Cronbach alpha values for the GAS was 0.948 .

\section{Children's Memory Questionnaire (CMQ)}

The CMQ is a 36-item questionnaire designed to assess parents' perceptions of their children's memory. The CMQ requires parents to assess their child's memory based on five possible options: $1=$ never or almost never happens; $2=$ happens less than once a week; $3=$ happens once or twice in a week; $4=$ happens about once a day; and $5=$ happens more than once a day [19]. Three subscales derived from the total scale representing the episodic memory, visual memory and working memory and attention. The higher the scores, the greater the impairment in the cognition domain [19]. In this study, the Cronbach alpha values for the episodic memory subscale was 0.888 , for the visual memory was 0.770 and for the working memory was 0.845 .

\section{Clinical attention problems scale}

The scale measures the frequency of activity and attention by asking the parent and teacher to respond to a series of 12 statements and their applicability to their child in the morning and afternoon. Response options range from "not true" (0), "somewhat or sometimes true" (1), "very often" or often true (2). The higher the scores, the greater the attention problems exist [20]. In this study, the Cronbach alpha values for the clinical attention problem in the morning and in the afternoon were 0.844 and 0.839 respectively.

\section{Learning, Executive and Attention Functioning (LEAF) Scale}

The LEAF is a 55 item self-report questionnaire that assesses executive functions, related neurocognitive functions, and academic skills in children and adults. The LEAF evaluates a broad set of core cognitive abilities as well as related cognitive learning and academic abilities. Cognitive areas assessed by the LEAF include attention, processing speed (including visual-spatial organization skills), and sustained sequential processing to achieve goals (e.g., planning and executing goal-directed behavior), working memory, and novel problem-solving. Also, LEAF includes comprehension and concept formation, declarative/factual memory, and academic functioning. The LEAF contains Academic subscales assessing reading, writing, and math fluency and abilities. LEAF items are grouped by subscale, and all subscales have the same number of items.

The subscales of the LEAF are: (1) comprehension and conceptual learning (tracking and understanding information), (2) factual memory (memorization and retention of facts); (3) attention (sustained focus); (4) processing speed (speed of completing cognitive and behavioral tasks that involve a component of focus and concentration); (5) visual-spatial organization (organization and visual-constructive skills); (6) sustained sequential processing (planning and sustaining effort in order to follow and complete multistep directions and sequences); (7) working memory (remembering and processing multiple things at the same time); and (8) novel problem solving (initiating effort toward processing new or unfamiliar information). (9) Mathematics skills (math calculation difficulty); (10) basic reading skills (reading/phonics difficulty); and (11) written expression skills (limited/impoverished or slow/effortful written expression). Individual items are rated on a $0-3$ scale, and a raw subscale score 
for each of the 11 content areas is created by summing the 5 constituent items, such that higher scores indicate more cognitive problems [21]. In this study, the Cronbach alpha values for the subscales was: comprehension and conceptual learning $=0.961$; factual memory $=0.792$; attention $=0.901$; processing speed $=0.866$; visual-spatial organization $=0.729$; sustained sequential processing $=0.768$; working memory $=0.816$; novel problem solving $=0.811$; mathematics skills $=0.871$; basic reading skills $=0.923$ and written expression skills $=0.905$.

\section{Translation procedure}

The forward translation was done by one translator. An expert committee formed by healthcare professionals and a language professional verified the Arabic translated version. A backward translation was then performed by a second translator, unaware of the initial English version. The back-translated English questionnaire was subsequently compared to the original English one, by the expert committee. Discrepancies related to inadequate expressions and concepts, confusing in meaning and slightly off in meaning during the reconciliation of the back translated questionnaire with the original source were resolved by consensus.

\section{Statistical analysis}

SPSS software version 23 was used to conduct data analysis. Cronbach's alpha values were recorded for reliability analysis for all the scales. A descriptive analysis was done using the counts and percentages for categorical variables and mean and standard deviation for continuous measures. A multivariate analysis of covariance (MANCOVA) was carried out to compare multiple measures (each scale was taken as a dependent variable) taking the GAS as the major independent variable, controlling for potential confounding variables: age, gender, family monthly income, and mother and father education level. A p-value less than 0.05 was considered significant.

\section{Results}

Out of 700 distributed questionnaires, 566 (80.86\%) questionnaires were completed and collected back. The sociodemographic characteristics of the participants are summarized in Table 1 . The mean age was $10.77 \pm 1.38$ years, with $55.2 \%$ male. Also, higher education level in parents was found in 58.9\% among mother and $39.4 \%$ among father.

\section{Description of the scales used}

The description of all the scales used in terms of mean, standard deviation, median, minimum and maximum is summarized in Table 2.

\section{Bivariate analysis}

The bivariate analysis of the sociodemographic variables associated with the memory and attention scores is summarized in Tables 3 and 4.

Higher addiction to video gaming salience, tolerance, relapse, withdrawal, conflict and problems scores were significantly correlated with lower/worse episodic

Table 1 Sociodemographic characteristics of the study sample

\begin{tabular}{lcr}
\hline & Frequency & Percentage (\%) \\
\hline Gender & & 55.2 \\
Male & 286 & 44.8 \\
Female & 232 & 2.9 \\
Education level father & 14 & 7.0 \\
Illiterate & 34 & 22.9 \\
Primary & 111 & 27.8 \\
Complementary & 135 & 39.4 \\
Secondary & 191 & \\
University & & 2.9 \\
Education level mother & 14 & 2.2 \\
Illiterate & 11 & 11.9 \\
Primary & 58 & 24.1 \\
Complementary & 118 & 58.9 \\
Secondary & 288 & SD \\
University & Mean & 1.38 \\
\hline
\end{tabular}


Table 2 Description of the scales used

\begin{tabular}{|c|c|c|c|c|c|}
\hline Scale & Mean & $\begin{array}{l}\text { Standard } \\
\text { deviation }\end{array}$ & Median & Minimum & Maximum \\
\hline Episodic memory & 6.32 & 7.06 & 4 & 0 & 46 \\
\hline Working memory & 3.99 & 5.45 & 2 & 0 & 32 \\
\hline Visual memory & 2.38 & 3.86 & 1 & 0 & 27 \\
\hline Clinical attention morning & 16.75 & 4.56 & 16 & 0 & 34 \\
\hline Clinical attention afternoon & 17.22 & 4.96 & 16 & 0 & 36 \\
\hline Comprehension conceptual learning & 2.14 & 2.78 & 1 & 0 & 15 \\
\hline Factual memory & 1.98 & 2.51 & 1 & 0 & 15 \\
\hline Attention & 2.56 & 3.34 & 1 & 0 & 15 \\
\hline Processing speed & 2.75 & 3.30 & 2 & 0 & 15 \\
\hline Visual spatial organization & 2.75 & 2.85 & 2 & 0 & 16 \\
\hline Sustained sequential processing & 2.74 & 2.81 & 2 & 0 & 14 \\
\hline LEAF working memory & 2.81 & 2.84 & 2 & 0 & 15 \\
\hline Novel problem solving & 2.50 & 2.72 & 2 & 0 & 15 \\
\hline Mathematical skills & 2.19 & 2.97 & 1 & 0 & 15 \\
\hline Basic reading skills & 2.64 & 3.30 & 1 & 0 & 15 \\
\hline Written expression skills & 3.53 & 3.55 & 3 & 0 & 15 \\
\hline Addiction video gaming salience & 3.95 & 3.56 & 3 & 0 & 12 \\
\hline Addiction video gaming tolerance & 3.85 & 3.56 & 3 & 0 & 12 \\
\hline Addiction video gaming mood modification & 5.00 & 3.95 & 4 & 0 & 12 \\
\hline Addiction video gaming relapse & 5.56 & 3.75 & 6 & 0 & 12 \\
\hline Addiction video gaming withdrawal & 4.16 & 3.29 & 4 & 0 & 12 \\
\hline Addiction video gaming conflict & 2.85 & 2.88 & 2 & 0 & 12 \\
\hline Addiction video gaming problems & 1.39 & 2.22 & 0 & 0 & 12 \\
\hline
\end{tabular}

Table 3 Bivariate analysis of factors associated with the memory and attention scores

\begin{tabular}{|c|c|c|c|c|c|c|c|c|c|}
\hline Variable & $\begin{array}{l}\text { Episodic } \\
\text { memory }\end{array}$ & $\begin{array}{l}\text { Working } \\
\text { memory }\end{array}$ & $\begin{array}{l}\text { Visual } \\
\text { memory }\end{array}$ & $\begin{array}{l}\text { Clinical } \\
\text { attention } \\
\text { morning }\end{array}$ & $\begin{array}{l}\text { Clinical } \\
\text { attention } \\
\text { afternoon }\end{array}$ & $\begin{array}{l}\text { Comprehension } \\
\text { conceptual } \\
\text { learning }\end{array}$ & $\begin{array}{l}\text { Factual } \\
\text { memory }\end{array}$ & Attention & $\begin{array}{l}\text { Processing } \\
\text { speed }\end{array}$ \\
\hline \multicolumn{10}{|l|}{ Gender } \\
\hline Male & $6.65 \pm 7.03$ & $4.42 \pm 5.80$ & $2.36 \pm 3.68$ & $17.14 \pm 4.65$ & $17.72 \pm 5.13$ & $2.26 \pm 3.01$ & $2.04 \pm 2.60$ & $2.82 \pm 3.60$ & $2.89 \pm 3.47$ \\
\hline Female & $5.84 \pm 7.13$ & $3.38 \pm 4.90$ & $2.33 \pm 3.97$ & $16.21 \pm 4.40$ & $16.55 \pm 4.64$ & $2.00 \pm 2.50$ & $1.85 \pm 2.34$ & $2.19 \pm 2.95$ & $2.52 \pm 3.05$ \\
\hline$p$ & 0.209 & 0.034 & 0.932 & 0.028 & 0.011 & 0.307 & 0.405 & 0.041 & 0.224 \\
\hline \multicolumn{10}{|c|}{ Educational level } \\
\hline $\begin{array}{c}\text { Illiterate/ } \\
\text { primary }\end{array}$ & $7.87 \pm 9.52$ & $6.15 \pm 7.80$ & $2.83 \pm 3.59$ & $17.70 \pm 5.39$ & $18.28 \pm 5.53$ & $3.32 \pm 3.37$ & $3.09 \pm 3.15$ & $4.15 \pm 4.38$ & $3.61 \pm 3.75$ \\
\hline $\begin{array}{l}\text { Comple- } \\
\text { mentary }\end{array}$ & $6.22 \pm 7.24$ & $4.81 \pm 5.64$ & $2.31 \pm 3.49$ & $17.19 \pm 4.35$ & $17.72 \pm 5.08$ & $2.56 \pm 2.87$ & $2.08 \pm 2.58$ & $3.16 \pm 3.63$ & $3.41 \pm 3.68$ \\
\hline Secondary & $5.52 \pm 6.43$ & $3.53 \pm 4.64$ & $2.14 \pm 3.79$ & $16.98 \pm 4.74$ & $17.25 \pm 4.74$ & $2.06 \pm 2.59$ & $2.00 \pm 2.36$ & $2.52 \pm 3.32$ & $2.95 \pm 3.39$ \\
\hline University & $6.28 \pm 6.53$ & $2.97 \pm 4.58$ & $2.10 \pm 3.52$ & $16.02 \pm 4.23$ & $16.66 \pm 4.72$ & $1.45 \pm 2.40$ & $1.52 \pm 2.28$ & $1.73 \pm 2.56$ & $1.95 \pm 2.68$ \\
\hline$p$ & 0.273 & 0.001 & 0.645 & 0.054 & 0.146 & $<0.001$ & 0.002 & $<0.001$ & $<0.001$ \\
\hline \multicolumn{10}{|c|}{ Mother's educational level } \\
\hline $\begin{array}{c}\text { Illiterate/ } \\
\text { primary }\end{array}$ & $4.50 \pm 4.05$ & $6.08 \pm 6.85$ & $3.58 \pm 4.48$ & $16.65 \pm 5.73$ & $17.83 \pm 5.16$ & $3.78 \pm 4.38$ & $3.09 \pm 3.28$ & $3.00 \pm 3.90$ & $4.09 \pm 4.57$ \\
\hline $\begin{array}{l}\text { Comple- } \\
\text { mentary }\end{array}$ & $6.65 \pm 7.67$ & $5.28 \pm 6.17$ & $2.89 \pm 3.32$ & $17.89 \pm 4.65$ & $18.52 \pm 5.49$ & $3.07 \pm 3.27$ & $2.94 \pm 3.20$ & $4.07 \pm 4.00$ & $3.93 \pm 3.35$ \\
\hline Secondary & $6.39 \pm 8.47$ & $3.92 \pm 5.66$ & $2.09 \pm 3.98$ & $16.75 \pm 4.90$ & $17.23 \pm 5.24$ & $2.06 \pm 2.61$ & $1.62 \pm 1.88$ & $2.37 \pm 3.05$ & $2.97 \pm 3.38$ \\
\hline University & $6.31 \pm 6.53$ & $3.47 \pm 4.88$ & $2.13 \pm 3.67$ & $16.53 \pm 4.26$ & $16.96 \pm 4.61$ & $1.74 \pm 2.40$ & $1.77 \pm 2.43$ & $2.29 \pm 3.21$ & $2.32 \pm 3.07$ \\
\hline$p$ & 0.639 & 0.025 & 0.162 & 0.262 & 0.187 & $<0.001$ & 0.001 & 0.003 & 0.001 \\
\hline
\end{tabular}

Italic values indicate significant $p$-values 
Table 4 Bivariate analysis of factors associated with the memory and attention scores

\begin{tabular}{|c|c|c|c|c|c|c|c|}
\hline Variable & $\begin{array}{l}\text { Visual spatial } \\
\text { organization }\end{array}$ & $\begin{array}{l}\text { Sustained } \\
\text { sequential } \\
\text { processing }\end{array}$ & $\begin{array}{l}\text { LEAF } \\
\text { working } \\
\text { memory }\end{array}$ & Novel problem & Mathematics skills & Basic reading skills & $\begin{array}{l}\text { Written } \\
\text { expression } \\
\text { skills }\end{array}$ \\
\hline \multicolumn{8}{|l|}{ Gender } \\
\hline Male & $2.96 \pm 3.00$ & $2.94 \pm 2.93$ & $2.94 \pm 3.09$ & $2.57 \pm 2.88$ & $1.97 \pm 2.78$ & $2.89 \pm 3.56$ & $3.76 \pm 3.82$ \\
\hline Female & $2.44 \pm 2.60$ & $2.45 \pm 2.62$ & $2.60 \pm 2.49$ & $2.36 \pm 2.48$ & $2.45 \pm 3.16$ & $2.24 \pm 2.80$ & $3.21 \pm 3.19$ \\
\hline$p$ & 0.046 & 0.059 & 0.194 & 0.413 & 0.086 & 0.026 & 0.09 \\
\hline \multicolumn{8}{|c|}{ Father's educational level } \\
\hline Illiterate/primary & $3.54 \pm 3.54$ & $3.60 \pm 3.44$ & $3.83 \pm 3.73$ & $3.08 \pm 3.23$ & $3.44 \pm 3.91$ & $4.02 \pm 4.15$ & $4.70 \pm 4.11$ \\
\hline Complementary & $3.25 \pm 3.26$ & $3.08 \pm 3.07$ & $3.21 \pm 3.00$ & $2.96 \pm 2.95$ & $2.42 \pm 3.10$ & $3.03 \pm 3.21$ & $4.25 \pm 3.79$ \\
\hline Secondary & $2.62 \pm 2.64$ & $2.82 \pm 2.93$ & $2.74 \pm 2.80$ & $2.63 \pm 2.74$ & $2.22 \pm 2.91$ & $2.60 \pm 3.29$ & $3.68 \pm 3.83$ \\
\hline University & $2.26 \pm 2.34$ & $2.19 \pm 2.24$ & $2.21 \pm 2.31$ & $1.92 \pm 2.40$ & $1.53 \pm 2.32$ & $1.92 \pm 2.78$ & $2.64 \pm 2.80$ \\
\hline$p$ & 0.006 & 0.006 & 0.001 & 0.005 & $<0.001$ & $<0.001$ & $<0.001$ \\
\hline \multicolumn{8}{|c|}{ Mother's educational level } \\
\hline Illiterate/primary & $3.59 \pm 3.81$ & $3.13 \pm 2.38$ & $3.41 \pm 2.97$ & $2.82 \pm 2.50$ & $2.73 \pm 2.86$ & $3.32 \pm 2.61$ & $3.64 \pm 3.50$ \\
\hline Complementary & $3.46 \pm 3.21$ & $3.77 \pm 3.58$ & $3.71 \pm 3.62$ & $3.70 \pm 3.47$ & $3.28 \pm 3.33$ & $2.91 \pm 2.84$ & $4.96 \pm 4.09$ \\
\hline Secondary & $2.96 \pm 3.01$ & $3.04 \pm 2.94$ & $2.57 \pm 2.58$ & $2.27 \pm 2.44$ & $2.19 \pm 3.32$ & $2.81 \pm 3.39$ & $3.52 \pm 3.63$ \\
\hline University & $2.45 \pm 2.53$ & $2.41 \pm 2.58$ & $2.62 \pm 2.72$ & $2.31 \pm 2.68$ & $1.85 \pm 2.63$ & $2.41 \pm 3.32$ & $3.23 \pm 3.37$ \\
\hline$p$ & 0.026 & 0.004 & 0.035 & 0.005 & 0.007 & 0.404 & 0.012 \\
\hline
\end{tabular}

Post hoc analysis: Father's education level: working memory (illiterate/primary vs secondary $p=0.021$; illiterate/primary vs university $p=0.002$; complementary vs university $p=0.026$ ); comprehension conceptual morning (illiterate/primary vs secondary $p=0.036$; illiterate/primary vs university $p<0.001$; complementary vs university $p=0.005$ ); Factual memory (illiterate/primary vs university $p=0.001$ ); attention (illiterate/primary vs secondary $p=0.022$; illiterate/primary vs university $p<0.001$; complementary vs university $p=0.003$ ); processing speed (illiterate/primary vs university $p=0.014$; complementary vs university $p=0.002$ ); visual spatial organization (illiterate/primary vs university $p=0.034$; complementary vs university $p=0.026$ ); sustained sequential processing (illiterate/primary vs university $p=0.014$ ); working memory (illiterate/primary vs university $p=0.003$; complementary vs university $p=0.024$ ); novel problem solving (complementary vs university $p=0.013$ ); mathematics skills (illiterate/primary vs university $p<0.001$ ); basic reading skills (illiterate/primary vs university $p<0.001$; complementary vs university $p=0.033$ ); written expression (illiterate/primary vs university $p=0.002$; complementary vs university $p=0.001$ ). Mother's education level: comprehension conceptual learning (illiterate/primary vs secondary $p=0.033$; illiterate/primary vs university $p=0.003$; complementary vs university $p=0.005$ ); Factual memory (complementary vs secondary $p=0.006$; complementary vs secondary $p=0.008$ ); LEAF attention (complementary vs secondary $p=0.012$; complementary vs university $p=0.002$ ); processing speed (complementary vs university $p=0.005$ ); sustained sequential processing (complementary vs university $p=0.005$ ); novel problem solving (complementary vs secondary $\mathrm{p}=0.009$; complementary vs secondary $\mathrm{p}=0.004$ ); mathematics skills (complementary vs university $\mathrm{p}=0.005$ ); written expression (complementary vs university $p=0.006$ )

Italic values indicate significant $p$-values

memory, working memory, visual memory, clinical attention in the morning and in the afternoon, comprehension and conceptual learning, factual memory, LEAF attention, processing speed, visual spatial organization, sustained sequential processing, LEAF working memory, novel problem solving, mathematics skills, basic reading and written expression skills scores, except for the mood modification that was not associated with the visual memory and mathematics skills scores (Table 5).

\section{Multivariate analysis}

The MANCOVA analysis was performed taking the scales as the dependent variables and the addiction to video gaming salience as the independent variable, adjusting for the covariates (age, gender, family monthly income, and mother and father education levels).

Higher addiction to video gaming salience was significantly associated with higher episodic memory score (worse episodic memory), higher novel problem solving score (worse problem solving), higher basic reading skills score (worse basic reading skills), higher written expression skills score (worse written expression skills) and higher clinical attention in the morning and afternoon scores (worse episodic memory and attention).

Higher addiction to video gaming tolerance were significantly associated with higher novel problem solving score (worse novel problem solving) and higher clinical attention in the morning and afternoon scores (worse attention).

Higher addiction to video gaming withdrawal were significantly associated with higher clinical attention in the afternoon score (worse attention), higher factual memory score (worse factual memory), higher attention score (worse attention), higher processing speed score (worse processing speed), higher visual spatial organization score (worse visual spatial organization), higher sustained sequential processing score (worse sustained sequential processing), higher working memory score (worse working memory), higher novel problem solving score (worse novel problem solving) and 
Table 5 Bivariate analysis of continuous variables associated ${ }^{d}$ with the video gaming addiction subscales scores

\begin{tabular}{|c|c|c|c|c|c|c|c|}
\hline Variable & $\begin{array}{l}\text { Addiction } \\
\text { video gaming } \\
\text { salience }\end{array}$ & $\begin{array}{l}\text { Addiction } \\
\text { video gaming } \\
\text { tolerance }\end{array}$ & $\begin{array}{l}\text { Addiction video } \\
\text { gaming mood } \\
\text { modification }\end{array}$ & $\begin{array}{l}\text { Addiction video } \\
\text { gaming relapse }\end{array}$ & $\begin{array}{l}\text { Addiction } \\
\text { video gaming } \\
\text { withdrawal }\end{array}$ & $\begin{array}{l}\text { Addiction } \\
\text { video gaming } \\
\text { conflict }\end{array}$ & $\begin{array}{l}\text { Addiction } \\
\text { video gaming } \\
\text { problems }\end{array}$ \\
\hline Episodic memory & $0.363^{c}$ & $0.353^{c}$ & $0.216^{c}$ & $0.263^{c}$ & $0.293^{c}$ & $0.325^{c}$ & $0.244^{c}$ \\
\hline Working memory & $0.250^{c}$ & $0.253^{c}$ & $0.169^{c}$ & $0.211^{c}$ & $0.298^{c}$ & $0.299^{c}$ & $0.312^{c}$ \\
\hline Visual memory & $0.137^{b}$ & $0.133^{b}$ & 0.069 & $0.119^{b}$ & $0.209^{c}$ & $0.274^{c}$ & $0.343^{c}$ \\
\hline $\begin{array}{l}\text { Clinical attention } \\
\text { morning }\end{array}$ & $0.361^{c}$ & $0.305^{c}$ & $0.278^{c}$ & $0.320^{c}$ & $0.343^{c}$ & $0.329^{c}$ & $0.232^{c}$ \\
\hline $\begin{array}{l}\text { Clinical attention } \\
\text { afternoon }\end{array}$ & $0.334^{c}$ & $0.283^{c}$ & $0.248^{c}$ & $0.300^{c}$ & $0.323^{c}$ & $0.300^{c}$ & $0.231^{c}$ \\
\hline $\begin{array}{l}\text { Comprehension } \\
\text { conceptual } \\
\text { learning }\end{array}$ & $0.201^{c}$ & $0.210^{c}$ & $0.154^{b}$ & $0.143^{b}$ & $0.242^{c}$ & $0.200^{c}$ & $0.226^{c}$ \\
\hline Factual memory & $0.204^{c}$ & $0.241^{c}$ & $0.138^{b}$ & $0.179^{c}$ & $0.296^{c}$ & $0.291^{c}$ & $0.258^{c}$ \\
\hline LEAF attention & $0.287^{c}$ & $0.277^{c}$ & $0.211^{c}$ & $0.228^{c}$ & $0.293^{c}$ & $0.246^{c}$ & $0.218^{c}$ \\
\hline Processing speed & $0.264^{c}$ & $0.253^{c}$ & $0.169^{c}$ & $0.210^{c}$ & $0.302^{c}$ & $0.245^{c}$ & $0.215^{c}$ \\
\hline $\begin{array}{l}\text { Visual spatial } \\
\text { organization }\end{array}$ & $0.337^{c}$ & $0.312^{c}$ & $0.210^{c}$ & $0.255^{c}$ & $0.338^{c}$ & $0.287^{c}$ & $0.173^{c}$ \\
\hline $\begin{array}{l}\text { Sustained } \\
\text { sequential } \\
\text { processing }\end{array}$ & $0.282^{c}$ & $0.284^{c}$ & $0.222^{c}$ & $0.282^{c}$ & $0.329^{c}$ & $0.222^{c}$ & $0.152^{b}$ \\
\hline $\begin{array}{l}\text { LEAF working } \\
\text { memory }\end{array}$ & $0.263^{c}$ & $0.243^{c}$ & $0.198^{c}$ & $0.213^{c}$ & $0.295^{c}$ & $0.235^{c}$ & $0.177^{c}$ \\
\hline $\begin{array}{l}\text { Novel solving } \\
\text { problem }\end{array}$ & $0.226^{c}$ & $0.185^{c}$ & $0.175^{c}$ & $0.164^{c}$ & $0.272^{c}$ & $0.218^{c}$ & $0.155^{b}$ \\
\hline $\begin{array}{l}\text { Mathematics } \\
\text { skills }\end{array}$ & $0.148^{b}$ & $0.133^{b}$ & 0.081 & $0.099^{a}$ & $0.140^{b}$ & $0.130^{b}$ & $0.160^{b}$ \\
\hline $\begin{array}{l}\text { Basic reading } \\
\text { skills }\end{array}$ & $0.270^{c}$ & $0.230^{c}$ & $0.150^{b}$ & $0.202^{c}$ & $0.232^{c}$ & $0.209^{c}$ & $0.160^{b}$ \\
\hline $\begin{array}{l}\text { Written expres- } \\
\text { sion }\end{array}$ & $0.310^{c}$ & $0.289^{c}$ & $0.191^{c}$ & $0.214^{c}$ & $0.293^{c}$ & $0.217^{c}$ & $0.107^{a}$ \\
\hline
\end{tabular}

${ }^{\mathrm{a}} p<0.05 ;{ }^{\mathrm{b}} p<0.01 ;{ }^{\mathrm{c}} p<0.001$

higher written expression skills score (worse written expression skills) (Table 6).

\section{Discussion}

Thus far, this is the first study to be conducted on Lebanese school children to evaluate the association between addiction to video games, memory, attention and learning abilities. Taking into consideration that intensive video gaming has frequently been linked to the development of addictive-like behaviors among children, we found highly important to assess some cognitive factors that could be affected in this delicate population. The results showed that higher addiction to video gaming was significantly associated with higher memory score (worse memory), with higher attention score (worse attention), and higher LEAF scale and subscales scores (worse cognitive and academic abilities). We also found that having a father with university education level was significantly associated with lower attention score (better attention) and lower LEAF scale and subscales scores (better cognitive and academic abilities).

Higher addiction to video gaming was significantly associated with higher memory score (worse memory). When the different studies were reviewed, the results were found to be contradictory. Some of the studies argued that video games have negative effects on memory, while other ones did not support this finding [22]. Moreover, an observational study comparing patients with internet gaming disorder against healthy control groups revealed that the formers had lower working memory functioning [8]. This particular result regarding memory may relate to the fact that spending too much time playing the same type of games could be harming their abilities to retain and boost memories.

Moreover, higher addiction to video gaming was significantly associated with higher attention score (worse attention). Similar to our results, increasing behavioral studies proved that video games addicts showed attention deficits. De facto, Zhou et al. [23] demonstrated that 
Table 6 Multivariate analysis of covariance (MANCOVA)

\begin{tabular}{|c|c|c|c|c|c|}
\hline & \multirow[t]{2}{*}{ Beta } & \multirow{2}{*}{$\begin{array}{l}\text { Partial Eta } \\
\text { squared }\end{array}$} & \multirow[t]{2}{*}{ p-value } & \multicolumn{2}{|c|}{$95 \%$ confidence interval } \\
\hline & & & & Lower bound & Upper bound \\
\hline \multicolumn{6}{|l|}{ Episodic memory } \\
\hline Addiction video gaming salience & 0.581 & 0.024 & 0.009 & 0.149 & 1.013 \\
\hline \multicolumn{6}{|l|}{ Working memory } \\
\hline Addiction video gaming salience & 0.292 & 0.013 & 0.058 & -0.010 & 0.594 \\
\hline Addiction video gaming mood modification & 0.213 & 0.012 & 0.061 & -0.010 & 0.437 \\
\hline Father university compared to illiteracy/primary level* & -1.886 & 0.012 & 0.070 & -3.929 & 0.157 \\
\hline Mother university compared to illiteracy/primary level* & -2.522 & 0.011 & 0.077 & -5.315 & 0.272 \\
\hline \multicolumn{6}{|l|}{ Visual memory } \\
\hline Mother secondary compared to illiteracy/primary level* & -2.204 & 0.024 & 0.009 & -3.851 & -0.558 \\
\hline Addiction video gaming withdrawal & 0.139 & 0.010 & 0.088 & -0.021 & 0.299 \\
\hline \multicolumn{6}{|l|}{ Clinical attention morning } \\
\hline Addiction video gaming salience & 0.562 & 0.057 & $<0.001$ & 0.294 & 0.830 \\
\hline Addiction video gaming tolerance & 0.411 & 0.026 & 0.007 & 0.114 & 0.708 \\
\hline Father university compared to illiteracy/primary level* & -2.052 & 0.017 & 0.027 & -3.866 & -0.238 \\
\hline \multicolumn{6}{|l|}{ Clinical attention afternoon } \\
\hline Addiction video gaming salience & 0.561 & 0.049 & 0.001 & 0.270 & 0.851 \\
\hline Addiction video gaming tolerance & 0.367 & 0.018 & 0.025 & 0.046 & 0.689 \\
\hline Addiction video gaming withdrawal & 0.293 & 0.016 & 0.035 & 0.020 & 0.565 \\
\hline \multicolumn{6}{|l|}{ LEAF comprehension conceptual learning } \\
\hline Father university compared to illiteracy/primary level $\left.\right|^{*}$ & -1.445 & 0.021 & 0.006 & -2.464 & -0.425 \\
\hline Mother university compared to illiteracy/primary level* & -1.348 & 0.017 & 0.055 & -2.723 & 0.028 \\
\hline \multicolumn{6}{|l|}{ LEAF factual memory } \\
\hline Addiction video gaming withdrawal & 0.156 & 0.019 & 0.019 & 0.026 & 0.286 \\
\hline Father university compared to illiteracy/primary level* & -1.103 & 0.011 & 0.015 & -1.988 & -0.218 \\
\hline Mother secondary compared to illiteracy/primary level* & -1.724 & 0.027 & 0.006 & -2.960 & -0.489 \\
\hline Mother university compared to illiteracy/primary level* & -1.357 & 0.026 & 0.026 & -2.551 & -0.164 \\
\hline \multicolumn{6}{|l|}{ LEAF attention } \\
\hline Addiction video gaming withdrawal & 0.172 & 0.012 & 0.043 & 0.005 & 0.339 \\
\hline Father university compared to illiteracy/primary level* & -1.660 & 0.021 & 0.014 & -2.988 & -0.332 \\
\hline \multicolumn{6}{|l|}{ LEAF processing speed } \\
\hline Addiction video gaming withdrawal & 0.245 & 0.023 & 0.011 & 0.057 & 0.434 \\
\hline \multicolumn{6}{|l|}{ LEAF visual spatial organization } \\
\hline Addiction video gaming withdrawal & 0.145 & 0.013 & 0.044 & 0.004 & 0.285 \\
\hline \multicolumn{6}{|l|}{ LEAF sustained sequential processing } \\
\hline Addiction video gaming withdrawal & 0.244 & 0.032 & 0.001 & 0.098 & 0.389 \\
\hline \multicolumn{6}{|l|}{ LEAF working memory } \\
\hline Addiction video gaming withdrawal & 0.183 & 0.022 & 0.014 & 0.037 & 0.329 \\
\hline Father university & -1.254 & 0.006 & 0.019 & -2.302 & -0.205 \\
\hline \multicolumn{6}{|l|}{ LEAF novel problem solving } \\
\hline Addiction video gaming salience & 0.212 & 0.025 & 0.008 & 0.056 & 0.369 \\
\hline Addiction video gaming tolerance & 0.214 & 0.021 & 0.016 & 0.041 & 0.388 \\
\hline Addiction video gaming withdrawal & 0.233 & 0.033 & 0.002 & 0.086 & 0.379 \\
\hline \multicolumn{6}{|l|}{ LEAF mathematic skills } \\
\hline Gender (females vs males*) & 0.849 & 0.020 & 0.018 & 0.148 & 1.551 \\
\hline Father secondary level of education compared to illiteracy/primary level* & -1.319 & 0.015 & 0.040 & -2.578 & -0.059 \\
\hline Father university level of education compared to illiteracy/primary level* & -1.534 & 0.021 & 0.015 & -2.769 & -0.299 \\
\hline \multicolumn{6}{|l|}{ LEAF basic reading skills } \\
\hline Addiction video gaming salience & 0.220 & 0.016 & 0.035 & 0.015 & 0.425 \\
\hline Father university level of education compared to illiteracy/primary level* & -1.919 & 0.026 & 0.007 & -3.305 & -0.533 \\
\hline LEAF written expression skills & & & & & \\
\hline
\end{tabular}


Table 6 (continued)

\begin{tabular}{|c|c|c|c|c|c|}
\hline & \multirow[t]{2}{*}{ Beta } & \multirow{2}{*}{$\begin{array}{l}\text { Partial Eta } \\
\text { squared }\end{array}$} & \multirow[t]{2}{*}{$p$-value } & \multicolumn{2}{|c|}{$95 \%$ confidence interval } \\
\hline & & & & Lower bound & Upper bound \\
\hline Addiction video gaming salience & 0.236 & 0.012 & 0.019 & 0.038 & 0.433 \\
\hline Addiction video gaming withdrawal & 0.231 & 0.016 & 0.015 & 0.044 & 0.417 \\
\hline Father university & -1.917 & 0.022 & 0.005 & -3.256 & -0.577 \\
\hline
\end{tabular}

*Reference group

when performing the attention-related task, more total error rates was observed in subjects with internet gaming addiction in comparison to controls. To boot, Song et al. [24] established a positive correlation between inattention and internet gaming addiction severity, while lack of attention was verified to be a high predictor of online gaming addiction by Ko et al. [25]. The finding we obtained could be interpreted by the instant rewards and constant stimulation of video games that raise the threshold for children to pay attention in less stimulating situations, where working harder is required to get rewards. Another theory could be that children suffering from attentional problems become captivated by video games as a coping strategy of their behavioral disorder.

Likewise, higher addiction to video gaming was significantly associated with higher LEAF scale and subscales scores (worse cognitive and academic abilities). Correspondingly, Gentile et al. [26] found that video games addiction among Singaporean adolescents predicted worse school grades and cognitive well-being. In contrast, another study conducted by Regina et al. [11] demonstrated no evidence that excessive video gaming negatively affects adolescents' school performances. Our result may be understood in the light of the fact that more time spent on video games means less time spent on academic activities, as well as less hours of sleep overall, which generally make children with heavy video gaming attitudes, less alert and more susceptible to cognitive errors.

In addition to the previous findings, having a father with university education level was significantly associated with lower attention score (better attention) and lower LEAF scale and subscales scores (better cognitive and academic abilities). To our knowledge, no study in the literature discussed the education level of the parents, particularly the father, in this particular context. The cause for our result could be that highly educated fathers raise their children in a similar educative way, which enhances their cognitive and academic skills.

\section{Clinical implications}

The current study provides many valuable contributions to the literature. Despite growing numbers of published studies examining cognitive skills in children playing video games, there is a paucity of rigorous ones from which to draw firm conclusions. This study procures firm evidence that addiction to video games is linked to worse memory, attention, as well as cognitive and academic skills in school children. This analysis constitutes a vital first step towards a better understanding of video games addiction in children, supporting its evidence as a plausible entity with detrimental association. By this, the study encourages parents of children addicted to video games to set particular gaming rules (such as limiting gaming time and avoiding exploration of new games), advocates schools to introduce children to other fun activities to do, and highlights the importance of consulting a therapist in extreme cases.

\section{Limitations}

There are several limitations that should be noted. First of all, all scales were self-rated, which may only show high risk of addiction to video gaming rather than the diagnosis. Second, residual confounding bias is also possible, since there could be factors related to video gaming such as pre-existing attention problems, ADHD diagnosis that were not measured in this study. Moreover, parents might have over-or underestimated the answers to the questions, possibly predisposing us to information bias. A selection bias is also possible because of the enrollment of the students from three schools only and because of the convenient sample. The group of students chosen in this study were not representative of most of students in their age. Students' parents filled up the video gaming addiction scale, which could lead to information bias since this scale is not applicable to them. To add on that, since this study is cross-sectional, the findings of this study cannot address the causal relationships among the primary constructs of interest. Finally, the scales used have not been validated in Lebanon. The use of a random sample is an important advantage to the study, thus, the results we obtained can be generalized to the whole population. The results cannot be generalized to the whole population since it only recruited students from private schools, as well as a high percentage of well-educated mothers. A prospective longitudinal study is needed to better follow up the cognitive function of children over time. 


\section{Conclusion}

In conclusion, the results suggest a correlation between addiction to video games and worse memory, attention, as well as cognitive and academic abilities among school children. Those findings indicate the need for more extensive research, and serve to highlight vital next steps needed in future papers, such as identifying predicting factors that could aid in early detection of video gaming addiction in children.

\section{Abbreviations}

DSM-5: Diagnostic and Statistical Manual of Mental Disorders; ICD-11: 11th Revision of International Classification of Diseases; ADHD: Attention deficit hyperactivity disorder; GASC: Game Addiction Scale for Children; CMQ: Children's Memory Questionnaire; LEAF: Learning, Executive and Attention Functioning Scale; MANCOVA: Multivariate analysis of covariance.
\end{abstract}

\section{Acknowledgements}

The authors would like to thank the mothers for accepting to be part of this study.

\section{Authors' contributions}

The authors would like to thank Mr. Jihad Gerges, Mr. Joseph Chahine and the following persons of College Central des Moines Libanais Maronites-Jounieh: Father Superior Antoine Salame, General Director Father Dr. Wadih Al Skayem, Mrs. Hélène Chbeir-Director of the kindergarten, Mr. Joseph Rassi-Director of the primary cycle as well as the teachers of the primary cycle for their help in the data collection. The authors would like to thank Pr. William G. Kronenberger for giving us the permission to use the LEAF scale. Special thanks to the parents who participated in this study and for Ms. Marine Arisdakessian for her help in the data entry. All authors read and approved the final manuscript.

\section{Funding}

None.

\section{Availability of data and materials}

There is no public access to all data generated or analyzed during this study to preserve the privacy of the identities of the individuals. The dataset that supports the conclusions is available to the corresponding author upon request.

\section{Ethics approval and consent to participate}

The Psychiatric Hospital of the Cross Ethics and Research Committee approved this study protocol (HPC-012-2018). A written consent was obtained from the students and from their parents prior to starting the data collection.

\section{Consent for publication}

Not applicable.

\section{Competing interests}

The authors declare that they have no competing interests.

\section{Author details}

${ }^{1}$ Faculty of Medicine and Medical Sciences, Holy Spirit University of Kaslik (USEK), Jounieh, Lebanon. ${ }^{2}$ Research and Psychology Departments, Psychiatric Hospital of the Cross, P.O. Box 60096, Jal Eddib, Lebanon. ${ }^{3}$ INSERM, Univ. Limoges, CH Esquirol, IRD, U1094 Tropical Neuroepidemiology, Institute of Epidemiology and Tropical Neurology, GEIST, Limoges, France. ${ }^{4}$ INSPECT-LB: Institut National de Santé Publique, Epidémiologie Clinique Et ToxicologieLiban, Beirut, Lebanon. ${ }^{5}$ Faculty of Arts and Sciences, Holy Spirit University of Kaslik (USEK), Jounieh, Lebanon. ${ }^{6}$ Faculty of Pharmacy, Lebanese University, Beirut, Lebanon. ${ }^{7}$ Faculty of Medicine, University of Nicosia, Nicosia, Cyprus.

Received: 4 June 2020 Accepted: 4 December 2020

Published online: 12 December 2020

\section{References}

1. Nielsen website. Multi-plateform gaming: for the win! https://www.niels en.com/us/en/insights/article/2014/multi-platform-gaming-for-the-win/.

2. Jo YS, Bhang SY, Choi JS, Lee HK, Lee SY, Kweon YS. Clinical characteristics of diagnosis for internet gaming disorder: comparison of DSM-5 IGD and ICD-11 GD diagnosis. J Clin Med. 2019;8(7):945.

3. Torres-Rodriguez A, Griffiths MD, Carbonell X, Oberst U. Internet gaming disorder in adolescence: psychological characteristics of a clinical sample. J Behav Addict. 2018;7(3):707-18.

4. Nogueira M, Faria H, Vitorino A, Silva FG, Serrao NA. Addictive video game use: an emerging pediatric problem? Acta Med Port. 2019;32(3):183-8.

5. Christakis DA. The challenges of defining and studying "digital addiction" in children. JAMA. 2019;321(23):2277-8.

6. Kok Eren $\mathrm{H}$, Orsal O. Computer game addiction and loneliness in children. Iran J Public Health. 2018;47(10):1504-10.

7. Choi BY, Huh S, Kim D-J, Suh SW, Lee S-K, Potenza MN. Transitions in problematic internet use: a one-year longitudinal study of boys. Psychiatry Investig. 2019:16(6):433-42.

8. Du X, Yang Y, Gao P, Qi X, Du G, Zhang Y, et al. Compensatory increase of functional connectivity density in adolescents with internet gaming disorder. Brain Imaging Behav. 2017;11(6):1901-9.

9. Lim JA, Lee JY, Jung HY, Sohn BK, Choi SW, Kim YJ, et al. Changes of quality of life and cognitive function in individuals with internet gaming disorder: a 6-month follow-up. Medicine. 2016;95(50):e5695.

10. Teng Z, Nie Q, Guo C, Zhang Q, Liu Y, Bushman BJ. A longitudinal study of link between exposure to violent video games and aggression in Chinese adolescents: the mediating role of moral disengagement. Dev Psychol. 2019;55(1):184-95.

11. van den Eijnden R, Koning I, Doornwaard S, van Gurp F, Ter Bogt T. The impact of heavy and disordered use of games and social media on adolescents' psychological, social, and school functioning. J Behav Addict. 2018;7(3):697-706.

12. Evren C, Evren B, Dalbudak E, Topcu M, Kutlu N. Relationships of internet addiction and Internet gaming disorder symptom severities with probable attention deficit/hyperactivity disorder, aggression and negative affect among university students. Atten Defic Hyperact Disord. 2019;11(4):413-21.

13. Stavropoulos V, Adams BLM, Beard CL, Dumble E, Trawley S, Gomez R, et al. Associations between attention deficit hyperactivity and internet gaming disorder symptoms: is there consistency across types of symptoms, gender and countries? Addict Behav Rep. 2019;9:100158.

14. Hawi NS, Samaha M, Griffiths MD. Internet gaming disorder in Lebanon: relationships with age, sleep habits, and academic achievement. J Behav Addict. 2018;7(1):70-8.

15. Obeid S, Saade S, Haddad C, Sacre H, Khansa W, Al Hajj R, et al. Internet addiction among Lebanese adolescents: the role of self-esteem, anger, depression, anxiety, social anxiety and fear, impulsivity, and aggressiona cross-sectional study. J Nerv Ment Dis. 2019;207(10):838-46.

16. Hawi NS, Samaha M. Validation of the Arabic version of the internet gaming disorder-20 test. Cyberpsychol Behav Soc Netw. 2017;20(4):268-72.

17. Hawi NS, Samaha M, Griffiths MD. The digital addiction scale for children: development and validation. Cyberpsychol Behav Soc Netw. 2019;22(12):771-8.

18. YIlmaz E, Griffiths MD, Kan A. Development and validation of videogame addiction scale for children (VASC). Int J Ment Health Addict. 2017;15(4):869-82.

19. Drysdale K, Shores A, Levick W. Use of the everyday memory questionnaire with children. Child Neuropsychol. 2004;10(2):67-75.

20. Edelbrock C, Rancurello M. Childhood hyperactivity: an overview of rating scales and their applications. Clin Psychol Rev. 1985;5(5):429-45.

21. Castellanos I, Kronenberger WG, Pisoni DB. Questionnaire-based assessment of executive functioning: psychometrics. Appl Neuropsychol Child. 2018;7(2):93-109.

22. Ozcetin M, Gumustas F, Cag Y, Gokbay IZ, Ozmel A. The relationships between video game experience and cognitive abilities in adolescents. Neuropsychiatr Dis Treat. 2019;15:1171-80.

23. Zhou Z, Li C, Zhu H. An error-related negativity potential investigation of response monitoring function in individuals with internet addiction disorder. Front Behav Neurosci. 2013;7:131. 
24. Song J, Park JH, Han DH, Roh S, Son JH, Choi TY, et al. Comparative study of the effects of bupropion and escitalopram on internet gaming disorder. Psychiatry Clin Neurosci. 2016;70(11):527-35.

25. Ko CH, Yen JY, Chen CS, Yeh YC, Yen CF. Predictive values of psychiatric symptoms for internet addiction in adolescents: a 2-year prospective study. Arch Pediatr Adolesc Med. 2009;163(10):937-43.

26. Gentile DA, Choo H, Liau A, Sim T, Li D, Fung D, et al. Pathological video game use among youths: a two-year longitudinal study. Pediatrics. 2011;127(2):e319-329.

\section{Publisher's Note}

Springer Nature remains neutral with regard to jurisdictional claims in published maps and institutional affiliations.
Ready to submit your research? Choose BMC and benefit from:

- fast, convenient online submission

- thorough peer review by experienced researchers in your field

- rapid publication on acceptance

- support for research data, including large and complex data types

- gold Open Access which fosters wider collaboration and increased citations

- maximum visibility for your research: over 100M website views per year

At BMC, research is always in progress.

Learn more biomedcentral.com/submissions 